\title{
Group-Theoretic Reduction of Latin Squares in Experimental Designs
}

\author{
ROBERTO N. PADUA \\ rnpadua@yahoo.com \\ Liceo de Cagayan University
}

Date Submitted: January 14, 2007

Final Revision Accepted: July 16, 2007

\begin{abstract}
A Latin square or Latin square design is an experimental design that controls nuisance factors by blocking them off in two directions in order to study the effect of a treatment variable. It is produced when each treatment appears once and only in each row and column of the square. In this study, group theory concepts are used to determine the subgroup of a group-structural Latin squares that would represent the original Latin square with minimal efficiency loss. Latin square of the order $6 \times 6$ to $12 \times 12$ that have group structures were considered. The largest subgroup for each Latin Square was determined by finding the maximal normal subgroup of the given group. Results revealed that, on the average, the efficiency loss was only $6 \%$.
\end{abstract}

Key words - Latin square, normal subgourp, factor group.

\section{INTRODUCTION}

Latin squares or the Latin square design is an experimental design that controls nuisance factors by blocking them off in two directions in order to study the effect of a treatment variable. Thus, a treatment variable, $T$, at three levels $A, B, C$ can be studied using a Latin square by controlling two nuisance factors $C$ and $R$, each at three levels $C_{1^{\prime}} C_{2^{\prime}} C_{3}$ and $R_{1^{\prime}} R_{2^{\prime}} R_{3^{\prime}}$ respectively. If the nuisance factor $C$ represents the columns and the nuisance factor $R$ the rows of a Latin square, then a $3 \times 3$ Latin square is produced when each of the treatment levels appears once and only once in each row and column(Montgomery, 1991).

A number of interesting mathematical questions arise out of Latin squares as 
described above. For instance, there is no known formula to determine the number of distinct Latin squares of size $n \times n$. Computer calculations, so far, has determined the number of distinct Latin squares up to $n=15$. A related question, which has some applications in high energy Physics, has to do with the saturation point of a Latin square. More precisely, what percent of the squares need to be known in order to construct a distinct Latin square? (Ref: http//mathworld.wolfram.com/LatinSquare. $\mathrm{html})$. The nature of questions related to Latin squares is such that even one without sophisticated background in mathematics can easily grasp. It is, therefore, a very alluring and seductive mathematical curiosity.

This study considers yet another dimension of Latin squares. Specifically, the study investigates the practical applicability of Latin squares in designing sub-optimal experiments with fewer treatments while preserving the original algebraic structure.

For a fixed $n$, all $n \times n$ Latin squares have the same mean squared errors (MSE) and are, therefore, equally efficient. Let:

$$
\text { (1) } \cdots R^{2}=\frac{S S T-S S E}{S S T}
$$

be a measure of efficiency of an experimental design, where SST stands for the total sum of squares, SSE stands for the error sum of squares. It is known that for a given $n$, all $n \times n$ Latin squares have the same $R^{2}$ value.

In many practical situations, fewer treatments (with at least the same level of efficiency) would be desirable since such would entail lesser costs and fewer experimental replications. From the standpoint of Latin square experimental designs, therefore, the logical question to ask would be: what choice of $m<n$ would meet a suboptimality criterion, e.g. minimal reduction in $R^{2}$ ? In addition, how should the $m<n$ fewer treatment be chosen?

In search for answer to these questions, one is inevitably led to the concepts found in group theory. A group $G$ is a non-empty set together with a binary operation * satisfying the following conditions: (a) there exists a unique element $e \in G$ such that $a^{*} e$ $=e^{*} a=a$; (b) to each element $a \in G$, there exists a unique element $b \in G$, such that $a^{*} b=$ $b^{*} a=e$; and (c) for all $a, b, c \in G,\left(a^{*} b\right)^{*} c=a^{*}\left(b^{*} c\right)$. In addition, if $a^{*} b=b^{*} a$ for all $a, b \in G$, then we say $\mathrm{G}$ is an abelian group. It is interesting to note that for a fixed $n$, some of the $n x$ $n$ Latin squares form a group structure while others do not.

Denote the class of $n x n$ Latin squares that form a group by $\Gamma_{n}$. If the number of Latin squares of size $n x n$ is $\left|\mathrm{L}_{n}\right|$, then it is clear that $\left|\Gamma_{n}\right| \leq\left|\mathrm{L}_{n}\right|$ with equality holding only for $n=2$. It is logical to begin the statistical analysis by considering an $n x n$ Latin square $G_{n} \in \Gamma_{\mathrm{n}}$. Since $G_{n}$ (of order $\mathrm{n}$ ) possesses an algebraic structure as mentioned and has the same efficiency given by (1) as all the other Latin squares in $\mathrm{L}_{\mathrm{n}^{\prime}}$, we consider the

subgroups $S_{m} \leq G_{n}$ and study their statistical properties in relation to the efficiency measure (1). Note that by Lagrange's theorem, $\left|S_{m}\right|$ divides $\left|G_{n}\right|$. (Fraleigh,1989).

Section 2 discusses the statistical concepts of Latin squares and distribution of quadratic forms in normal random variables. Section 3 presents some useful concepts in finite group theory. Section 4 gives some analyses of the group structure of Latin squares. Section 5 investigates the theoretical connections between finite groups and the statistical properties of Latin squares belonging to $\Gamma_{n}$. Section 6 provides some numerical results related to the problem through simulation. 


\section{Statistical Concepts and Latin Squares}

The most commonly used random variables in practice are those coming from a normal distribution with mean $\mu$ and variance $\sigma^{2}$. Let $x_{1}, x_{2} \ldots x n$ be independent and identically distributed random variables from a normal distribution with mean $\mu$ and variance $\sigma^{2}$, we symbolize this as:

(2) $\ldots x_{1}, x_{2}, \ldots, x_{n}{ }^{i i d} \sim N\left(\mu, \sigma^{2}\right)$.

Theorem 1 summarizes the distribution of random variables which are functions of the x's.

$$
\text { iid }
$$

Theorem 1. Let $x_{1}, x_{2}, \ldots, x_{n} \sim N\left(\mu, \sigma^{2}\right)$, then :

$$
\begin{aligned}
& 1.1 \bar{X}=\frac{\sum x_{i}{ }^{d}}{n} N\left(\mu, \frac{\sigma^{2}}{n}\right) \\
& 1.2 Z=\frac{\bar{x}-\mu}{\frac{\sigma}{\sqrt{n}}} \stackrel{d}{\sim} N(0,1)
\end{aligned}
$$

1.3 If $Z_{1}, Z_{2}, \ldots, Z_{n}$ are iid $N(0,1)$ then $y=Z_{1}^{2}+Z_{2}^{2}+\ldots+Z_{n}^{2}$ has a $\sigma^{2}$ chi-square distribution with $n$ degrees of freedom.

Proof: (See Mood, Graybill and Boes, 1975).

If a random variable $X$ has a chi-square distribution with $m$ degrees of freedom, and $\mathrm{y}$ is an independent chi-square random variable with $n$ degrees of freedom, thenth ratio $f=\frac{x / m}{y / n}$ has an F-distribution with $m$ and $n$ degrees of freedom.

The results of theorem 1 are easily extended to multivariate normal vectors. A random $p$-component vector $X$ has a $p$-variate normal distribution with mean vector $\mu$ and covariance matrix $\Sigma$ if its density can be written in the form:

$$
\text { (3) } \ldots f(x)=\frac{1}{(2 \pi)^{\frac{p}{2}}|\Sigma|^{\frac{1}{2}}} \exp \left[-\frac{1}{2}(x-\mu)^{\prime} \Sigma^{-1}(x-\mu)\right]
$$

provided that the inverse of $\Sigma$ exists. The $p$-variate standard normal distribution, therefore, has the density:

$$
\text { (4) } \ldots f(Z)=\frac{1}{(2 \pi)^{\frac{p}{2}}} \exp \left(-\frac{1}{2} Z^{\prime} Z\right)
$$

(Theorem 2 summarizes the results relative to multivariate normal random vectors.

Theorem 2. Let $X \sim^{d} M V N(\mu, \Sigma)$. Then : 
1.1 $Z=(X-\mu)^{\prime} \Sigma^{-1}(X-\mu)$ has a chi-square distribution with $p$ degrees of freedom;

2.2 If $Z \sim^{d} M V N(0,1)$, then if $A$ is a symmetric idempotent matrix of rank $p$, then $Z^{\prime} A Z$ has a chi-square distribution with $p$ degrees of freedom.

2.3 $Z^{\prime} A Z$ and $Z^{\prime} B Z$ are two quadratic forms in normal random vector $Z$, then the two are independent if $A B=0$.

Proof: (see Graybill, 1976).

A direct application of these results can be seen in the theory of general linear models (GLM). To this end, let:

$$
\text { (4) } \ldots y=x \beta+\varepsilon
$$

where $\mathrm{y}$ is a $n x 1$ observed random vector, $X$ is an $n x p$ matrix of constants, $\beta$ is a $p \times 1$ vector of unknown parameters and $\varepsilon$ is an $n \times 1$ random error assumed to obey a multivariate normal distribution with mean $\underline{0}$ and covariance matrix $\sigma^{2} I$. It is easy to verify that the maximum likelihood estimators of $\beta$ and $\sigma^{2}$ are:

$$
\begin{aligned}
& \text { (6) } \ldots \hat{\beta}=\left(x^{\prime} x\right)^{-1} x^{\prime} y \\
& \text { (7) } \ldots \hat{\sigma^{2}}=\frac{(y-\hat{y})^{\prime}(y-\hat{y})}{n-p}
\end{aligned}
$$

By an application of Theorem 1 and 2, we see that $\hat{\beta}$ has a normal distribution, $\operatorname{MVN}\left(\beta, \sigma^{2}\left(X^{\prime} X\right)^{-1}\right)$, and $\hat{S}^{2}$ has a chi-square distribution with $(n-p)$ degrees of freedom. It is instructive to analyze the numerator of (7). The following identities are easy to verify:

8) $\ldots(y-\bar{y})=(y-\hat{y}+\hat{y}-\bar{y})$

and : $\quad(y-\bar{y})(y-\bar{y})=(y-\hat{y})(y-\hat{y})+(\hat{y}-\bar{y})(\hat{y}-\bar{y})$

$$
S S T=S S E+S S R
$$

where:

$$
\begin{aligned}
S S T=(y-\bar{y}) & (y-\bar{y}) \\
S S E & =(y-\hat{y})(y-\hat{y}) \\
S S R & =(\hat{y}-\bar{y})(\hat{y}-\bar{y}) .
\end{aligned}
$$


The decomposition (8) is called the orthogonal decomposition of the total sum of squares into the error sum of squares (SSE) and the regression sum of squares (SSR). follows:

The decomposition (8) is often summarized in an analysis of variance table as

\begin{tabular}{|c|c|c|c|c|}
\hline Source of Errors & Sum of Squares & $\begin{array}{l}\text { Degrees of } \\
\text { Freedom }\end{array}$ & Mean Squares & F-ratios \\
\hline Regression & $S S R$ & $p-1$ & SSR & \\
\hline Error & SSE & $n-p$ & $M S E=\frac{\begin{array}{r}p-1 \\
S S E\end{array}}{n-p}$ & $F=\frac{M S R}{M S E}$ \\
\hline Total & SST & $n-1$ & & \\
\hline
\end{tabular}

In order to verify that the ratio called $\mathrm{f}$ is indeed F-distributed, it is necessary to check that the numerator and denominator are each independently chi-square distributed:

Theorem 3: In the standard GLM, the distribution of

$$
\begin{aligned}
& 3.1 \operatorname{SSE}=(y-\hat{y})^{\prime}(y-\hat{y}) \text { is } \sigma^{2} \chi_{n-p}^{2} \\
& 3.2 \operatorname{SSR}=(\hat{y}-\bar{y})^{\prime}(\hat{y}-\bar{y}) \text { is } \sigma^{2} \chi_{p-1}^{2}
\end{aligned}
$$

and the two are statistically independent.

Proof: We have :

3.1 Let $\hat{y}=x \hat{\beta}=x\left(x^{\prime} x\right)^{-1} x^{\prime} y=H y$ where $H=x\left(x^{\prime} x\right)^{-1} x^{\prime}$ is a symmetric idempotent matrix.

$$
\operatorname{SSE}=(y-\hat{y})^{\prime}(y-\hat{y})=y^{\prime}(I-H)(I-H) y=y^{\prime}(I-H) y
$$

since $(I-H)^{2}=I-H$ is also symmetric and idempotent. It follows from Theorem 2 that SSE has a chi-square distribution (actually $\sigma^{2} \chi_{n-p}^{2}$ ) with degrees of freedom equal to $\operatorname{rank}(I-H)=n-p$.

3.2 The proof for (3.2) is similar. It remains to show that (3.1) and (3.2) are independent sum of squares. To do this, note that :

$$
\hat{\varepsilon}=(y-\hat{y})=(y-x \hat{\beta})=\left(y-x\left(x^{\prime} x\right)^{-1} x^{\prime} y\right)=(I-H) y
$$

While

$$
\hat{y}=x \hat{\beta}=x\left(x^{\prime} x\right)^{-1} x^{\prime} y=H y
$$

Using Theorem 2, we note that:

$$
H(I-H)=\left(H-H^{2}\right)=(H-H)=0
$$


so that $\hat{y}$ and $\hat{\varepsilon}$ are orthogonal and so are SSE and SSR.

It follows from Theorem 3 that:

$$
\begin{aligned}
& \text { (9) } \ldots \text { MSE }=\frac{S S E}{n-p} \stackrel{d}{\sim} \sigma^{2} \chi_{n-p}^{2} \\
& \text { (10) } \ldots \text { MSR }=\frac{S S R}{p-1} \stackrel{d}{\sim} \sigma^{2} \chi_{p-1}^{2}
\end{aligned}
$$

and so:

$$
\text { (11) } \ldots \quad f=\frac{M S R}{M S E} \stackrel{d}{\sim} F(p-1, n-p) \text {. }
$$

\section{Application to Latin Squares}

The orthogonal decomposition (8) is a basic tool in Experimental Designs, particularly, for Latin square designs. The statistical model for a Latin square is:

$$
y_{i j k}=\mu+\alpha_{i}+\tau_{j}+\beta_{k}+\varepsilon_{i j k}\left\{\begin{array}{l}
i=1,2, \ldots, p \\
j=1,2, \ldots, p \\
k=1,2, \ldots, p
\end{array}\right\}
$$

While $y_{i j k}$ on the $i t h$ row, $k$ th column for the $j$ th treatment, $\mu$ is the overall mean, $\alpha$ is the $i$ th row effect, $\tau_{\mathrm{j}}$ is the $j$ th treatment effect and $\beta_{k}$ is the $k t h$ column effect. The row and column effects are the nuisance factors. The analysis of variance decomposition gives:

(13) $S S T=S S($ Row $)+S S($ Column $)+S S($ treatment $)+S S$ (Error $)$

with respective degrees of freedom:

$$
p^{2}-1=(p-1)+(p-1)+(p-1)+(p-2)(p-1) .(\text { Montgomery, 1991). }
$$

The treatment sum of squares (SS(treatment)) is obtained by collecting the data under each of the Latin letters (treatment), i.e. collect and add all data for treatment $A$, do the same for treatment $B$ and so on.

It follows from this observation that:

Theorem 4. For a fixed $n_{0}$ and a set of experimental data for $n_{0} x n_{0}$ Latin square design, any choice of $n_{0} x n_{o}$ Latin square gives the same error sum of squares.

Proof: The orthogonal decomposition applies regardless of the position of the Latin letter in the Latin square, hence, the totals for each Latin square will be the same. 


\section{Finite Groups and subgroups}

Let $G_{n}$ be a group of order $n$. Such a group that can be written on a group table is often done in abstract algebra.

Definition: Let $G$ be a group and let $H$ be a subset of $G$. Then, $H$ is a subgroup of $G$ if $H$ itself is a group under the same operation on $G$.

Remark: To verify that $H$ is a subgroup of $G$, it is only necessary to check that $e \in H$ and for all $a, b \in H$, then $a^{*} b^{-1} \in H$.

A group of order 2 has only the trivial subgroups $\{e\}$ and $G$ itself. Similarly, a group $G$ of order 3 has no proper non-trivial subgroups. We state without proof the famous Lagrange's Theorem:

Theorem 5. (Lagrange) The order of a subgroup $H$ divides the order of a group $G$.

The contra-positive of the theorem is useful for our present paper, namely: "If the order of $H$ does not divide the order of $G$, then $H$ is not a subgroup of $G$ ". Thus, groups of order 2 and 3 have no proper non-trivial subgroups. In fact,

Theorem 6. Every group of prime order $p$ has no proper non-trivial subgroups.

Proof: If $p$ is prime, then the only divisors of $p$ are 1 and $p$. Any other positive integer less than $p$ cannot divide $p$ and, hence, cannot be the order of subgroup of $H$.

Definition: Let $\left(G^{*}\right)$ and $\left.G^{\prime}, \mathrm{o}\right)$ be two groups of the same order. $G$ and $G^{\prime}$ are isomorphic to each other if there exists a function $\phi: G \rightarrow G^{\prime}$ that is one-to one and onto and satisfy:

$$
\Phi(\boldsymbol{a} * \boldsymbol{b})=\boldsymbol{\Phi}(\boldsymbol{a}) \boldsymbol{o} \boldsymbol{\Phi}(\boldsymbol{b}) \quad \forall a, b \in G
$$

Two groups are isomorphic if they are structurally the same. Obviously, two groups of different orders are not isomorphic. All groups of order 2 are isomorphic; similarly all groups of order 3 are isomorphic. A very important theorem in algebra relates groups of finite orders to the groups of permutations. We now state without proof, the following theorem.

Theorem 7: (Cayley) Every group of finite order is isomorphic to a subgroup of the group of permutations on $n$ letters.

\subsection{Groups and Latin Squares}

The purpose of this section is to analyze the group structures of $n x n$ Latin squares. Some of the results presented are new results while others are standard results in algebra. We first define terms to standardize our terminologies:

Definition: A finite table label is an arrangement of the Latin letters $\{A, B, C, \ldots, Z\}$ on the column and row headings. The labels are said to be the standard labels if they are arranged in their natural order.

\begin{tabular}{ccc} 
& The following are standard labels of $2 \times 2,3 \times 3$ finite tables: \\
\hline$*$ & $\mathrm{~B}$ \\
\hline $\mathrm{A}$ & $\mathrm{C}$ \\
$\mathrm{B}$ & \\
$\mathrm{C}$ & \\
\hline
\end{tabular}


The symbol "** is placed on the tables to denote a binary operation. This binary operation takes a row symbol and combines it with a column symbol to produce a symbol to be placed in the row and column intersections. If the resultant symbols are symbols coming from the row and column symbols and each symbol occurs once and only once in each row and column, then we have a Latin square.

Definition: Latin squares having standard labels are called labeled Latin squares. Our interest lies on the characterization of these labeled Latin squares for any $n$. For $n$ $=3$, there are $123 \times 3$ Latin squares but only 3 of these are groups. Theorem 5 below characterizes these group-structured Latin squares.

Theorem 8. (Diangca) A labeled $3 \times 3$ Latin square is a group if the first row and first column entries are identical and the entries are precisely the cyclic permutation of the standard sequence $(A B C)$, namely $(B C A)$ and $(C A B)$.

\begin{tabular}{ccc}
\hline$*$ & A & B \\
\hline A & & \\
B & \\
\hline
\end{tabular}

Proof: First notes that once the first row and first column of a $3 \times 3$ Latin square are known, then the rest of the entries are determined by the Latin square property. Consider the labeled Latin square whose first row and first column entries are $(A B C)$. It follows that $A$ is the identity element of this structure by symmetry. Consider the second row entries that begin with $B$. By Latin square property, $B^{*} B$ $=C$ and $B^{*} C=A$. Also for the third row entries that begin with $C$, we have $C^{*} B=$ $A$ and $C^{*} C=B$. It follows that $A^{-1}=A, B^{-1}=C$ and $C^{-1}=B$. The associative property follows by tedious calculation.

The proof for the tables with $(B C A)$ and $(C A B)$ as first row, first column entries is similar.

All the three $3 \times 3$ Latin groups are cyclic, that is, there is an element $x$ such that $x^{3}=$ $e$, the identity element for each of these groups. In fact,

Theorem 9. Every group of order 3 is cyclic, hence abelian.

Proof. Let $G$ be a group of order 3. Without loss of generality, let $\underline{e} \in G$ be the identity and let $A, B \neq e$ be the other element. Suppose that none of $A$ or $B$ generates $G$, then $A^{2}=e$ and $B^{2}=e$. Thus $A^{*} B \neq e$. Since $A^{*} e=A$, it follows that $A^{*} B=B$ from which $A$ $=e$, a contradiction.

If the leading entries (first row, first column) do not belong to the cyclic permutation of $(A B C)$, then the Latin square so formed does not possess a group structure. For instance, the table whose heading entries are $(C B A)$ does not form a group (it does not have an identity element) as one can easily verify. The presence of the sequence $(A B C)$, in that order, in any row or column of the table identifies the identity element (identity permutation). 


\begin{tabular}{cccc}
\hline$*$ & $\mathrm{~A}$ & $\mathrm{~B}$ & $\mathrm{C}$ \\
$\mathrm{A}$ & $\mathrm{A}$ & $\mathrm{B}$ & $\mathrm{C}$ \\
$\mathrm{B}$ & $\mathrm{B}$ & $\mathrm{C}$ & $\mathrm{A}$ \\
$\mathrm{C}$ & $\mathrm{C}$ & $\mathrm{A}$ & $\mathrm{B}$ \\
\hline
\end{tabular}

Figure Q

\begin{tabular}{llll}
\hline$*$ & $\mathrm{~A}$ & $\mathrm{~B}$ & $\mathrm{C}$ \\
$\mathrm{A}$ & $\mathrm{C}$ & $\mathrm{A}$ & $\mathrm{B}$ \\
$\mathrm{B}$ & $\mathrm{A}$ & $\mathrm{B}$ & $\mathrm{C}$ \\
$\mathrm{C}$ & $\mathrm{B}$ & $\mathrm{C}$ & $\mathrm{A}$ \\
\hline
\end{tabular}

Figure $\mathrm{S}$

\begin{tabular}{cccc}
\hline$*$ & $\mathrm{~A}$ & $\mathrm{~B}$ & $\mathrm{C}$ \\
$\mathrm{A}$ & $\mathrm{B}$ & $\mathrm{C}$ & $\mathrm{A}$ \\
$\mathrm{B}$ & $\mathrm{C}$ & $\mathrm{A}$ & $\mathrm{B}$ \\
$\mathrm{C}$ & $\mathrm{A}$ & $\mathrm{B}$ & $\mathrm{C}$ \\
\hline
\end{tabular}

Figure T

Figure T

In figure $Q$, the sequence $(A B C)$ appears on the first row, first column, hence $A$ is the identity element; in Figure $S$ the sequence $(A B C)$ appears on the third row, third column, hence the identity is $C$; in Figure $T$, it appears on the second row, second column, hence the identity is $B$.

As of this writing, we have not yet formulated a necessary and sufficient condition for a $4 \times 4$ labeled Latin square to be a group. However,

Theorem 10. If a labeled $4 x 4$ Latin square arises as a cyclic permutation of the standard sequence $(A B C D)$, then it is a group. Moreover, the identity element of the group is the label of the row (column) where the standard sequence appears.

Theorem 10 ensures that there are at least four (4) groups of labeled $4 x 4$ Latin squares.

Corollary 1. If a labeled $n \times n$ Latin square arises as a standard sequence $(A B C D \ldots)$, then it is a group.

Theorem 11. If an $n x n$ labeled Latin square is standard and the elements in each row or column are cyclic permutations of the letters, then $G$ is abelian.

Proof: Since every cyclic group is abelian (Fraleigh, 1989), it suffices to show that $G$ is cyclic. Let $\mathrm{A}_{\mathrm{j}}$ be the elements of Latin square. $\mathrm{j}=\{1,2,3, \ldots, n\}$ and $G=\left\{\rho_{\mathrm{i}}: \mathrm{i}=1,2, \ldots n\right.$ 1 ) where $\rho_{i}=$ action of every row or column on the $n$ elements of Latin square. Consider a Latin squares with cyclic permutation of $n$ elements. The $i$ th row or $j t h$ column of the Latin square gives the permutation

$$
\begin{gathered}
\rho_{i}: A_{j} \propto A_{(j+i)} \\
\rho_{1}=A_{j} \propto A_{j+1}, \\
\rho_{1}^{2}=\rho_{1} \rho_{1}: A_{j} \propto A_{j+i} \propto A_{j+2}=\rho_{2} \\
\rho_{1}^{3}=\rho_{1} \rho_{1}^{2}: A_{j} \propto A_{j+i} \propto A_{j+3}=\rho_{3},
\end{gathered}
$$




$$
\begin{aligned}
& \rho_{1}^{n-1}=\rho_{n-1} \\
& \rho_{1}^{0}=\rho_{0} \\
& \text { So } \rho_{1} \text { is the generator of } G . \\
& \left\langle\rho_{1}\right\rangle=G,
\end{aligned}
$$

Therefore, $G$ is cyclic, hence abelian.

Let $N_{\mathrm{G}}$ be the number of groups from $n \times n$ labeled Latin squares, it follows that $N_{G} \geq n$ from the Corollary.

\subsection{Sub-optimal Properties of Subgroups of Labeled Latin Squares.}

We begin the statistical analysis of group $n \times n$ Latin squares starting with $n=6$ since no proper non-trivial subgroups exist for $n=2$ and $n=3$. For $n=4$, the only possible subgroups would have order $m=2$ while a $2 \times 2$ Latin square is not possible. Also when $n$ is a prime, there is no proper non-trivial subgroups existing.

\subsection{Subgroups of $6 \times 6$ Group Labeled Latin Squares.}

$$
\begin{aligned}
& \text { Let: } y_{i j k}=\mu+\alpha_{i}+\tau_{j}+\beta_{k}+\varepsilon_{i j k}\left\{\begin{array}{l}
i=1,2,3,4,5,6 \\
j=1,2,3,4,5,6 \\
k=1,2,3,4,5,6
\end{array}\right\} \\
& \text { And: } y_{i . .}=\sum_{j} \sum_{k} y_{i j k} \quad y_{. . k}=\sum_{i} \sum_{j} y_{i j k} \quad y_{. j .}=\sum_{i} \sum_{k} y_{i j k} \\
& y_{. . .}=\sum_{i} \sum_{j} \sum_{k} y_{i j k}
\end{aligned}
$$

\begin{tabular}{|c|c|c|c|c|c|c|c|}
\hline & $\mathrm{A}$ & B & $\mathrm{C}$ & D & $\mathrm{E}$ & $\mathrm{F}$ & Totals \\
\hline A & $\mathrm{A}=1$ & $\mathrm{~B}=2$ & $\mathrm{C}=1$ & $\mathrm{D}=1$ & $\mathrm{E}=1$ & $\mathrm{~F}=1$ & 7 \\
\hline B & $B=-1$ & $C=-3$ & $D=-2$ & $E=-2$ & $F=-1$ & $A=-2$ & -11 \\
\hline C & $\mathrm{C}=2$ & $\mathrm{D}=1$ & $\mathrm{E}=2$ & $F=-2$ & $A=2$ & $B=-3$ & 2 \\
\hline D & $\mathrm{D}=-3$ & $E=-2$ & $\mathrm{~F}=1$ & $A=2$ & $\mathrm{~B}=3$ & $\mathrm{C}=-2$ & -1 \\
\hline E & $E=2$ & $F=-3$ & $A=2$ & $\mathrm{~B}=3$ & $\mathrm{C}=-2$ & $\mathrm{D}=2$ & 4 \\
\hline $\mathrm{F}$ & $\mathrm{F}=3$ & $A=-1$ & $B=-2$ & $\mathrm{C}=3$ & $\mathrm{D}=1$ & $\mathrm{E}=3$ & 7 \\
\hline Totals & 4 & -6 & 2 & 5 & 4 & -1 & 8 \\
\hline
\end{tabular}

be the standard notation for experimental designs. For convenience, we illustrate our point with an example using a $6 x 6$ Latin square experiment. Note that $6 x 6$ is the smallest Latin square dimension for which a sub-optimal subgroup Latin square $(3 \times 3)$ can be generated, i.e. a $2 \times 2$ Latin square is not feasible since the experimental error term cannot be estimated or the degrees of freedom is zero for SSE for this configuration.

Table 1. Coded data for a $6 \times 6$ latin square

The sum of squares can be easily computed as:

$\begin{array}{lllr}S S(\text { total }) & =154.222 & \text { SS }(\text { row }) & =38.222 \\ S S(\text { column }) & =14.555 & \text { SS } \text { treatment })= & 4.555 \\ S S(\text { error }) & =96.89 & & \end{array}$


with the corresponding Analysis of Variance table

Table 2. ANOVA table for the $6 x 6$ latin square experimental data

\begin{tabular}{lcccc}
\hline \multicolumn{1}{c}{ Sources of Errors } & Sum of Squares & $\begin{array}{c}\text { Degrees of } \\
\text { Freedom }\end{array}$ & Mean Square & f-ratio \\
\hline Row & 38.222 & 5 & & \\
Column & 14.555 & 5 & 0.7512 & $\mathrm{f}=0.1880$ \\
Treatment & 4.555 & 5 & 4.8445 & \\
Error & 96.89 & 20 & $R^{2}=37.17 \%$ & \\
\hline \multicolumn{1}{c}{ Total } & 154.222 & 35 & &
\end{tabular}

It is easily verified that the corresponding labeled $6 x 6$ Latin square forms a group with $A=e$, the identity. The set $H=\{A, C, E\}$ forms a subgroup of this group. The corresponding subgroup data are:

\begin{tabular}{cccc}
\hline $\mathrm{A}=1$ & $\mathrm{C}=1$ & $\mathrm{E}=1$ & 3 \\
$\mathrm{C}=2$ & $\mathrm{E}=2$ & $\mathrm{~A}=2$ & 6 \\
$\mathrm{E}=2$ & $\mathrm{~A}=2$ & $\mathrm{C}=-2$ & 2 \\
5 & 5 & 1 & 11 \\
\hline
\end{tabular}

Table 3. Data for the $3 \times 3$ Latin square subgroup.

Totals

The corresponding ANOVA Table for this configuration is given below.

Table 4. ANOVA for the $3 \times 3$ Latin square subgroup

\begin{tabular}{lcccc}
\multicolumn{5}{c}{ Table 4. ANOVA for the 3x3 Latin square subgroup } \\
\hline Sources of Errors & Sum of Squares & $\begin{array}{c}\text { Degrees of } \\
\text { Freedom }\end{array}$ & Mean Square & f-ratio \\
\hline Row (Reduced) & 3.889 & 2 & & \\
Column (Reduced) & 3.556 & 2 & 1.778 & $\mathrm{f}_{0}=1.002$ \\
Treatment (Reduced) & 3.556 & 2 & 1.775 & \\
Error (Reduced) & 3.555 & 2 & & \\
\hline \multicolumn{1}{c}{ Total (Reduced) } & 13.556 & \multicolumn{2}{c}{8} &
\end{tabular}

$$
R_{0}^{2}=73.78 \%
$$

Our interest lies in the distribution of the ratios of the $R^{2}$ value for reduced and original models. In this particular case, the ratio is:

$$
\phi=\frac{R_{T}^{2}}{R_{0}^{2}}=\frac{37.17}{73.78}=50.38 \%
$$

The ratio of the Mean Squared Error is:

$$
\gamma=\frac{M S E_{T}}{M S E_{0}}=\frac{4.8445}{1.775}=2.729
$$



, then

Toanalyzethedistributionof thesesratios, recallthatif $x_{1}, x_{2}, \ldots, x_{p} \sim N\left(\mu, \sigma^{2}\right)$

(17) $\ldots y=\frac{\left(x_{1}-\bar{x}\right)^{2}+\left(x_{2}-\bar{x}\right)^{2}+\ldots+\left(x_{p}-\bar{x}\right) 2}{\sigma^{2}} \sim \chi_{p-1}^{2}$

Consider the subset $\left(x_{q_{1}}, x_{q_{2}}, \ldots x_{q_{m}}\right), q_{m}<p$ where sample mean is $\bar{x}_{q}$. Then

(18) $\ldots Z=\frac{\left(x_{q_{1}}-\overline{x_{q}}\right)^{2}+\ldots+\left(x_{q_{m}}-\overline{x_{q}}\right) 2}{\sigma^{2}} \sim \chi_{q_{m}-1}^{2}$.

However, the variables $y$ and $z$ are not independent, i.e. SSE (full) and SSE (reduced) are not independent errors. In fact,

\begin{tabular}{cccc}
\hline $\mathrm{A}=1$ & $\mathrm{C}=1$ & $\mathrm{E}=1$ & 3 \\
$\mathrm{C}=2$ & $\mathrm{E}=2$ & $\mathrm{~A}=2$ & 6 \\
$\mathrm{E}=2$ & $\mathrm{~A}=2$ & $\mathrm{C}=-2$ & 2 \\
5 & 5 & 1 & 11 \\
\hline
\end{tabular}

(19) $\ldots E(y z)=2(q m-1)$

Upon noting that for $\mathrm{i} \neq \mathrm{j}$. It follows that the distribution of $\{\gamma \mathrm{i}\}$ cannot be an Fdistribution.

To remedy the situation, consider the random variables $(y-z)$ and $z$. Since

$\mathrm{E}(\mathrm{y}-\mathrm{z})(\mathrm{z})=\mathrm{E}(\mathrm{yz})-\mathrm{E}(\mathrm{z} 2)=\mathrm{E}(\mathrm{z} 2)-\mathrm{E}(\mathrm{z} 2)=0$, then $\mathrm{y}-\mathrm{z}$ and $\mathrm{z}$ are independent. Therefore, the ratio:

(20) ...f $f=\frac{\operatorname{SSE}(f u l l)-\operatorname{SSE}(\mathrm{red})}{\operatorname{SSE}(\mathrm{full})} \cdot \frac{\left(q_{m}-1\right)}{\left(p-q_{m}\right)}$

has an F-distribution with degrees of freedom $(p-1)-\left(q_{m}-1\right)$, and $\left(q_{m}-1\right)$ or $\left(p-q_{m}\right)$ and $\left(q_{m}-1\right)$ degrees of freedom.

From the preceding example, we have:

(21) $\ldots f=\left(\frac{96.89-3.555}{96.89}\right)\left(\frac{3-1}{6-3}\right)=0.6422$

can be compared with $\mathrm{F}_{.05}(3,2)=19.16$. We conclude that the error reduction is not significant. That is, the decrease in the error sum of squares due to reduction in the treatments is not statistically significant and the reduced model is accepted.

The subgroup $H=\{A, C, E\}$ is such that any binary combination of any two letters results in one of the three letters only (by the closure property). For instance, $A^{*} C=C$ and $C^{*} E=A$, the combined effect of $C$ and $E$ is the same as treatment $A$. In other words, the three treatments $A, C$ and $E$ represent redundant treatments, and it is possible to derive one treatment from the other, much like the original group structure for the $6 \mathrm{x}$ 6 Latin square.

The results indicate an increase in $R^{2}$ (from $37.17 \%$ to $73.70 \%$ ) and a decrease in the 
experimental error (from 4.8445 to 1.775 ). It is of interest to ask if the same technique can be done in general.

To put the results on a firm foundation, we define the concept of a factor group from a normal subgroup $H$ of $G$.

Definition. Let $H$ be a subgroup of $G$. Then, $H$ is normal subgroup of $G$ if $g H=H g \quad \forall$

$g \in G$. If $H$ is normal subgroup of $G$, then the set of cosets $\{g H\}$, denoted by $G \mid H$, forms a group under the induced operation on the coset called a factor group. For instance in the previous Latin square group $G$ of order 6, the subgroup $H=$ $\{A, C, E\}$ is normal in $G$. The factor group $G \mid H$ consists exactly of the elements $\{H, b+H, d+H, f+H\}$. Note that this factor group reduces to $\{H, b+H\}$ since $b+H=$ $d+H=f+H$. The factor group, in this case has cardinality 2. Since there is no $2 \times 2$ Latin square, we conclude that the subgroup $H=\{A, C, E\}$ represents the maximal compression of the original 6x6 Latin square, which preserves the group structure of the treatments.

Let $H_{1}$ and $H_{2}$ be normal subgroups of $G$. If $\left|H_{2}\right|>\left|H_{1}\right|$, then it follows that $|G|\left|H_{2}\right|<|G| H_{1} \mid$. Between the two subgroups, it is better to choose $H_{2}$ in the context of Latin square reduction since $G \mid H_{1}$ preserves more of the original treatments. In particular, if $|G| H \mid=2$, then $H$ represents the maximal compression of the treatments, which preserves the group structure.

Theorem 12. Let $H$ be a subgroup of $G$. If $|G| H \mid=2$, then $H$ is normal in $G$. Moreover, $H$ represents the maximal compression of the original treatment preserving the original group structure.

Proof: Suppose $g \in H$, since $H$ is a subgroup of $G \Rightarrow g H=H$ also $H g=H \Rightarrow g H=$ $H g$. Suppose $g \notin H \Rightarrow g \in H_{1} \Rightarrow g H_{1} \notin H \Rightarrow g H_{1}=H_{1}$ and $H_{1} g=H_{1} \Rightarrow g H_{1}=$ $H_{1} g$. Thus, $g H=H g$.

Consider a $12 x 12$ Latin square that forms a group. Let $H_{1}, H_{2}, H_{3}$, and $H_{4}$ be normal subgroups of order $2,3,4$, and 6 respectively. It follows that:

$$
\begin{aligned}
& G \mid H_{1} \cong H_{4} \\
& G \mid H_{2} \cong H_{3} \\
& G \mid H_{3} \cong H_{2} \\
& G \mid H_{4} \cong H_{1}
\end{aligned}
$$

The maximal treatment compression is $G\left|H_{1}\right| \cong \boldsymbol{H}_{4}$ for the original $12 x 12$ Latin square.

\section{Application Setting}

The general theory developed here can be used in the following setting. There is an experimental set-up where $n$ treatments are used. The treatments are $a b$ initio related to one another through a structure similar to groups. For example, if $A=$ use of cooperative learning, $B=$ use of laboratory method, $C=$ inductive approach, the $A^{*} B$ is the inductive approach $C$. We seek to reduce the number of treatments while preserving the original group structure. 


\section{Simulation}

In practice, computer simulation is done to analyze problems that are very difficult to determine analytically. In statistics, several assumptions are made for a certain situation before a probability model can be constructed. Analyzing the model usually involves computer simulation.

In this paper, a simulation program was developed in order to determine whether a subgroup that has a maximal compression of the original treatments will represent the original Latin square maintaining the group structure of the original Latin square. The simulation program used the formula

$$
y_{i j k}=\mu+\alpha_{i}+\tau_{j}+\beta_{k}+\varepsilon_{i j k}\left\{\begin{array}{l}
i=1,2, \ldots, p \\
j=1,2, \ldots, p \\
k=1,2, \ldots, p
\end{array}\right\}
$$

for a $p \times p$ Latin square, where $y_{i j k}$ were the data entries for each cell in a Latin square. The parameters $\mu, \alpha, \tau$, and $\beta$ had an assigned assumed values, while $\varepsilon_{i j k}$ took the values from a $N(0,1)$ generated random numbers.

The ratio of the measure of efficiency of the reduced and original Latin squares,

$$
\phi=\frac{F D_{\text {opriginal }}}{F D_{\text {reduced }}} \text { where } F D_{\text {original }} \text { and } F D_{\text {reduced }}
$$

is a measure of efficiency for original and reduced Latin squares respectively and their values were computed using equation (1). The F-ratio was computed using equation (20).

The Sum-of-Squares computing formulas (Montgomery,1991) are as follows:

$$
\begin{aligned}
& S S T=\sum_{i} \sum_{j} \sum_{k} y_{i j k}^{2}-\frac{y_{\ldots}^{2}}{n^{2}} \\
& S S(\text { Column })=\frac{\sum_{k} y_{. . k}^{2}}{n}-\frac{y_{\ldots}^{2}}{n^{2}}
\end{aligned}
$$$$
S S(\text { Rows })=\frac{\sum_{i} y_{i . .}^{2}}{n}-\frac{y_{\ldots}^{2}}{n^{2}}
$$

$$
S S(\text { Treatment })=\sum_{j} \frac{y_{. j .}^{2}}{n}-\frac{y_{\ldots}^{2}}{n^{2}}
$$

The group Latin square used in $6 \times 6,8 \times 8,9 \times 9,10 \times 10$, and $12 \times 12$ was in standard label. The first row and first column of the Latin square were standard (see appendix 1). The subgroups chosen were the largest subgroup for each group Latin square. In a $p x p$ Latin square where $p$ was even, the largest subgroup chosen was $m x m$ where $m$ was $p$ divided by 2 . The reduced (subgroup) Latin square was formed by choosing all odd rows and all odd columns respectively. For the $9 x 9$ Latin square, the reduced (subgroup) Latin square was formed by choosing the $1^{\text {st }}$ row and $1^{\text {st }}$ column, the $4^{\text {th }}$ row and $4^{\text {th }}$ column, and the $7^{\text {th }}$ row and $7^{\text {th }}$ column.

\section{Simulation Output}

In Table 6 on the following page, the value of $\mu$ was assumed at 3 , while the values of $\alpha, \tau$, and $\beta$ were placed in arrays consisting of different values. The simulation results for 1000 runs showed the average ratios of $F D$ of the original Latin squares, and their 
corresponding reduced Latin square were almost equal to 1 . Starting from the original $6 x 6$ to the original $12 \times 12$ Latin squares with their corresponding reduced Latin square, the $F D$ average ratio was more than 0.9 . This average ratio showed that the measures of efficiency of both the original and reduced Latin squares were almost equal. Their average f-ratios were almost equal, which was 0.6 except for $9 x 9$ Latin square which was 0.3 , but all their f-ratios were less than 1 .

Table 6. Simulation Results: The values of $\alpha, \tau$, and $\beta$ are

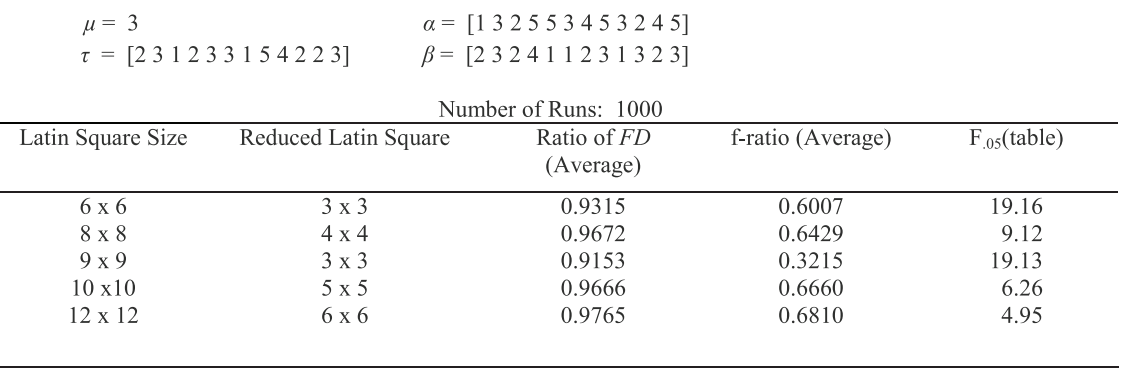

Fixing all values of $\alpha, \tau$, and $\beta$ equal to 1 , as shown in Table 7 on the following page, results in an average ratios of almost equal to 0.6 for all the 5 Latin squares. This means that the reduced Latin squares had greater values or greater efficiency compared to the originals. There was no significant change in the f-ratios compared to the f-ratio in Table 6.

Table 7. Simulation Results: Fixed values of $\alpha, \tau$, and $\beta$

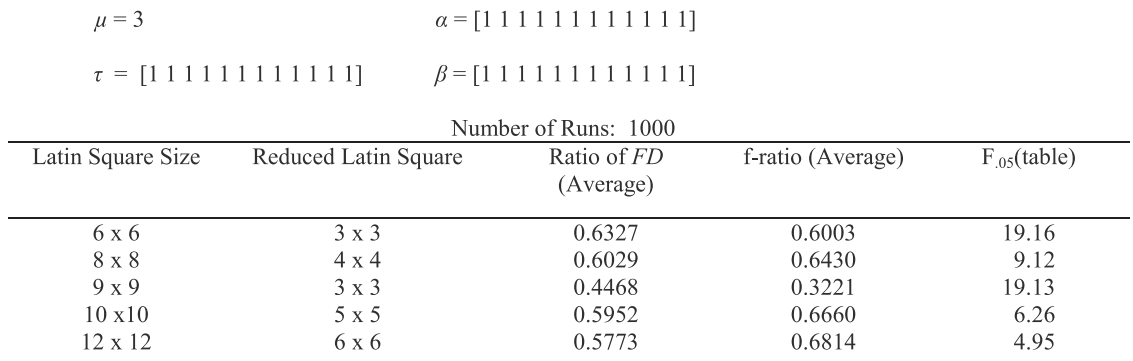

In Tables 8, 9, and 10, one parameter was fixed at 1 . The results show that the average $F D$ ratios for all the Latin squares listed were almost equal to 1 , while there was no significant change in their f-ratios.

Table 8. Simulation Results: Fixed value of $\alpha$

$$
\begin{array}{lll}
\mu=3 & \alpha=\left[\begin{array}{lllllllllll}
1 & 1 & 1 & 1 & 1 & 1 & 1 & 1 & 1 & 1 & 1
\end{array}\right] \\
\tau=\left[\begin{array}{lllllllllll}
2 & 3 & 1 & 2 & 3 & 3 & 1 & 5 & 4 & 2 & 2
\end{array}\right] & \beta=\left[\begin{array}{llllllllllll}
2 & 3 & 2 & 4 & 1 & 1 & 2 & 3 & 1 & 3 & 2 & 3
\end{array}\right]
\end{array}
$$


Number of Runs: 1000

\begin{tabular}{ccccr}
\hline Latin Square Size & Reduced Latin Square & $\begin{array}{c}\text { Ratio of } F D \\
\text { (Average) }\end{array}$ & f-ratio (Average) & $\mathrm{F}_{.05}($ table) \\
\hline $6 \times 6$ & $3 \times 3$ & 0.9368 & 0.5998 & 19.16 \\
$8 \times 8$ & $4 \times 4$ & 1.0500 & 0.6407 & 9.12 \\
$9 \times 9$ & $3 \times 3$ & 0.9301 & 0.3216 & 19.13 \\
$10 \times 10$ & $5 \times 5$ & 0.9574 & 0.6646 & 6.26 \\
$12 \times 12$ & $6 \times 6$ & 0.9979 & 0.6831 & 4.95
\end{tabular}

Table 9. Simulation Results: Fixed $\tau$

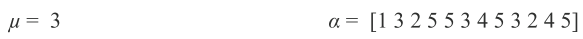

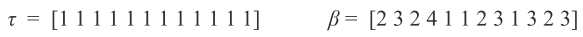

Number of Runs: 1000

\begin{tabular}{ccccr}
\hline Latin Square Size & Reduced Latin Square & Ratio of $F D$ (Average) & f-ratio (Average) & $\mathrm{F}_{.05}($ table) \\
& & & & \\
\hline $6 \times 6$ & $3 \times 3$ & 0.9252 & 0.6013 & 19.16 \\
$8 \times 8$ & $4 \times 4$ & 0.9336 & 0.6432 & 9.12 \\
$9 \times 9$ & $3 \times 3$ & 0.8604 & 0.3215 & 19.13 \\
$10 \times 10$ & $5 \times 5$ & 0.9576 & 0.6660 & 6.26 \\
$12 \times 12$ & $6 \times 6$ & 0.9725 & 0.6806 & 4.95 \\
\end{tabular}

Table 10. Simulation Results: Fixed $\beta$

\begin{tabular}{|c|c|c|c|c|}
\hline \multicolumn{5}{|c|}{$\alpha=\left[\begin{array}{llllllllllll}1 & 3 & 2 & 5 & 5 & 3 & 4 & 5 & 3 & 2 & 4 & 5\end{array}\right]$} \\
\hline \multicolumn{2}{|c|}{$\tau=\left[\begin{array}{llllllllllll}2 & 3 & 1 & 2 & 3 & 3 & 1 & 5 & 4 & 2 & 2 & 3\end{array}\right]$} & 11111111 & & \\
\hline \multicolumn{5}{|c|}{ Number of Runs: 1000} \\
\hline Latin Square Size & Reduced Latin Square & $\begin{array}{c}\text { Ratio of } F D \\
\text { (Average) }\end{array}$ & f-ratio (Average) & $\mathrm{F}_{.05}($ table $)$ \\
\hline $6 \times 6$ & $3 \times 3$ & 0.8939 & 0.6000 & 19.16 \\
\hline $8 \times 8$ & $4 \times 4$ & 0.9436 & 0.6423 & 9.12 \\
\hline $9 \times 9$ & $3 \times 3$ & 0.8960 & 0.3215 & 19.13 \\
\hline $10 \times 10$ & $5 \times 5$ & 0.9357 & 0.6676 & 6.26 \\
\hline $12 \times 12$ & $6 \times 6$ & 0.9465 & 0.6807 & 4.95 \\
\hline
\end{tabular}

In Tables 11, 12, and 13, two parameters were fixed at value equal to 1 . The results show that the average FD ratios were almost similar to those in Tables 8,9, and 10, which FD ratios were almost equal to 1 . The f-ratios again had no significant change.

Table 11. Simulation Results: Fixed $\alpha$ and $\tau$

$$
\begin{array}{llll}
\mu=3 & & \alpha=\left[\begin{array}{llllllllllll}
1 & 1 & 1 & 1 & 1 & 1 & 1 & 1 & 1 & 1 & 1 & 1
\end{array}\right] \\
\tau= & {\left[\begin{array}{lllllllllllll}
1 & 1 & 1 & 1 & 1 & 1 & 1 & 1 & 1 & 1 & 1 & 1
\end{array}\right]} & \beta=\left[\begin{array}{lllllllllll}
2 & 3 & 2 & 4 & 1 & 1 & 2 & 3 & 1 & 3 & 2
\end{array}\right]
\end{array}
$$


Number of Runs: 1000

\begin{tabular}{ccccr}
\hline $\begin{array}{c}\text { Latin Square Size } \\
\text { (Table 11 Continued) }\end{array}$ & Reduced Latin Square & $\begin{array}{c}\text { Ratio of } F D \\
\text { (Average) }\end{array}$ & f-ratio (Average) & $\mathrm{F}_{.05}($ table) \\
\hline $6 \times 6$ & $3 \times 3$ & 0.9979 & 0.5979 & 19.16 \\
$8 \times 8$ & $4 \times 4$ & 1.0700 & 0.6434 & 9.12 \\
$9 \times 9$ & $3 \times 3$ & 0.7689 & 0.3207 & 19.13 \\
$10 \times 10$ & $5 \times 5$ & 1.1055 & 0.6669 & 6.26 \\
$12 \times 12$ & $6 \times 6$ & 1.1565 & 0.6801 & 4.95 \\
\hline
\end{tabular}

Table 12. Simulation Results: Fixed $\tau$ and $\beta$

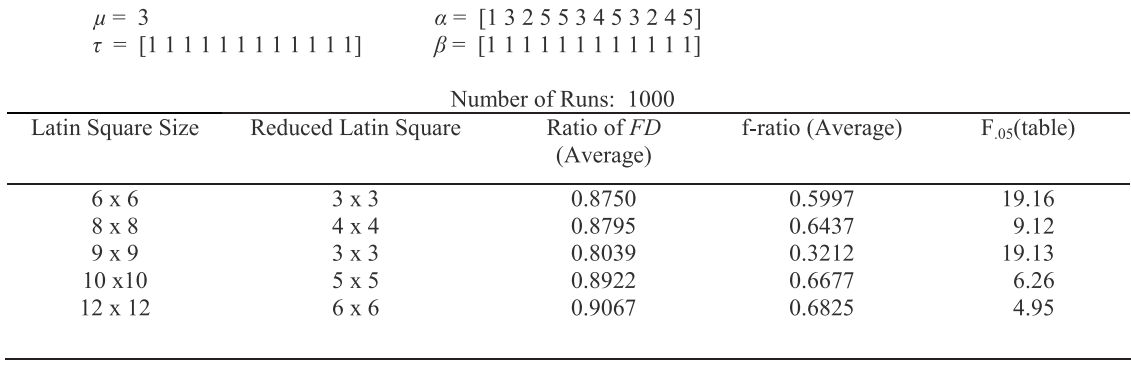

Table 13. Simulation Results: Fixed $\alpha$ and $\beta$

\begin{tabular}{|c|c|c|c|c|}
\hline $\begin{array}{l}\mu=3 \\
\tau=\left[\begin{array}{llll}2 & 3 & 1\end{array}\right]\end{array}$ & $1542223]$ & $\begin{array}{llllllll}1 & 1 & 1 & 1 & 1 & 1 & 1 & 1 \\
1 & 1 & 1 & 1 & 1 & 1 & 1\end{array}$ & & \\
\hline & & ff Runs: 1000 & & \\
\hline Latin Square Size & Reduced Latin Square & $\begin{array}{c}\text { Ratio of } F D \\
\text { (Average) }\end{array}$ & f-ratio (Average) & $\mathrm{F}_{.05}($ table $)$ \\
\hline $6 \times 6$ & $3 \times 3$ & 0.7600 & 0.5999 & 19.16 \\
\hline $8 \times 8$ & $4 \times 4$ & 0.9904 & 0.6429 & 9.12 \\
\hline $9 \times 9$ & $3 \times 3$ & 0.9983 & 0.3216 & 19.13 \\
\hline $10 \times 10$ & $5 \times 5$ & 0.9058 & 0.6647 & 6.26 \\
\hline $12 \times 12$ & $6 \times 6$ & 0.9055 & 0.6816 & 4.95 \\
\hline
\end{tabular}

\section{CONCLUSIONS}

The average values of the ratio of $F D$ between the original Latin square and its reduced Latin square were computed from $6 x 6$ up to $12 \times 12$ originals. Values of the parameters $\alpha, \tau$, and $\beta$ were assumed and $\mu$ was given a fixed value of 3 . The values of the error $\varepsilon$ came from the $N(0,1)$ random numbers. Changing the values of the parameters did not make any significant change in the values of f-ratios, which was less than 1.

From the simulation results, the reduced model of the Latin square, which was subgroup of the original Latin square, was as good as the original. The measure of the reduced efficiency was much greater than the original due to a decrease in experimental error of the reduced Latin square. This error was caused by a reduced number of treatments. Thus, selecting a specific object from a small sample size has lesser error than 
selecting a specific object from a larger sample size given that the object is in the sample size. For the $9 \times 9$ group Latin square, its corresponding subgroup has smaller sum of square error because its subgroup or reduced Latin square was only one third of the original; that is, it contains smaller number of treatments. Where as for the other group Latin squares its subgroup or reduced Latin square was only one half of the originals. The computed f-ratios were very small, making the reduced Latin squares as good as the original Latin squares. Therefore, the reduced Latin square, which represents the maximal compression of the original treatments, can be used in experimental designs to replace the original Latin squares for as long as the treatments are related to each other and are similar to a group structure. Using the reduced Latin square will reduce the number of treatments as well as lower the cost of the experiments.

\section{LITERATURE CITED}

Fraleigh, J. B. (1989). A First Course in Abstract Algebra, $4^{\text {th }}$ edition. Massachussets: Addison-Wesley Publishing Company.

Graybill, F. A. (1976). Theory and Application of the Linear Model. California: Wadsworth Publishing Company, Inc..

Montgomery, D. C. (1991). Design and Analysis of Experiments, $3^{\text {rd }}$ edition. New York: John Wiley and Sons.

Mood, A. M., Graybill, F. A., and Boes, D. A. (1974). Introduction to the theory of Statistics, $3^{\text {rd }}$ edition. New York: McGraw-Hill Inc. 